\title{
Detection of water ice grains after the DEEP IMPACT onto Comet 9P/Tempel 1
}

\author{
R. Schulz ${ }^{1}$, A. Owens ${ }^{2}$, P. M. Rodriguez-Pascual ${ }^{3}$, D. Lumb ${ }^{2}$, C. Erd ${ }^{2}$, and J. A. Stüwe ${ }^{4}$
}

1 ESA Research and Scientific Support Department, ESTEC, Postbus 299, 2200 AG Noordwijk, The Netherlands e-mail: rschulz@rssd.esa.int

2 ESA Science Payloads and Advanced Concepts Office, ESTEC, Postbus 299, 2200 AG Noordwijk, The Netherlands

3 ESA, XMM-Newton Science Operations Centre, ESAC, Apartado 50727, 28080 Madrid, Spain

4 Sterrewacht Leiden, Postbus 9513, 2300 RA Leiden, The Netherlands

e-mail: stuwe@strw.leidenuniv.nl

Received 28 September 2005 / Accepted 11 January 2006

\section{ABSTRACT}

Context. Icy grains in the inner coma of a comet may play an important role in the energy balance and in the production of certain gas coma species. Their existence has therefore been assumed repeatedly to explain a variety of observed phenomena. However, owing to their extremely short life time no evidence for the presence of icy grains had been found in any active comet close to the Sun.

Aims. We observed Comet 9P/Tempel 1 during the Deep Impact mission to look for phenomena induced by the impact.

Methods. The comet was observed with the XMM-Newton Observatory. We used the EPIC camera for X-ray imaging and the Optical Monitor for monitoring in the ultraviolet and visible spectral range.

Results. An outburst of the comet nucleus was observed as a result of the impact and the evolution of the coma was monitored in gas and dust. Our observations led to the first detection of icy grains in a comet at 1.5 AU from the Sun.

Conclusions. We showed for the first time that the material ejected from the nucleus of a comet contains icy grains, even at small heliocentric distance.

Key words. comets: general

\section{Introduction}

Ever since Fred Whipple (1950) developed the "dirty snowball" model to describe the nucleus of a comet, it has been suspected that an active nucleus would not only release pure gas and dust particles, the latter dragged out by the sublimating volatiles, but also "icy grains" composed of either pure ice or of dust particles covered with an ice mantle (Huebner \& Weigert 1966). Other possibilities include dust particles with ices embedded in them in interstitial regions or dust particles covered by complex tar-like hydrocarbon coatings. Knowledge of whether icy grains do indeed exist in the near-nucleus region of a comet is very important, because their existence has been proposed to explain a number of observed phenomena (see Hanner 1981, for a summary). Nevertheless, water ice grains had never been detected in any comet close to the Sun which can be attributed at least in part to their very short lifetime at small heliocentric distances. Hanner (1981) discussed the detectability of icy grains in the coma of comets finding that their detection is extremely difficult inside a heliocentric distance of around $2 \mathrm{AU}$, because the icy component sublimates within 1-2 $\mathrm{h}$ forming a secondary source for water and its decay products. Therefore, the search for icy grains was expected to be most promissing at large heliocentric distance, where the lifetime of such grains would be long enough for a icy grain halo to develop which might be observable at infrared wavelengths. Another reason for the non-detection of icy grains may be that under nominal conditions of a moderately active nucleus close to the Sun most of the material leaving the surface is indeed in the form of either dust or gas. Only when the nucleus is being eroded very quickly in very active comets or during outbursts, would we expect to see both, non-volatile dust particles and "icy grains" of any of the above mentioned possible types. Therefore, to find evidence for the possible existence of icy grains in the inner coma of a comet, one has to observe at a time, when a large amount of icy grains is produced under non-steady state conditions, for instance during an outburst.

On 4 July 2005, 5:52 UT the DEEP IMPACT mission impacted a $370 \mathrm{~kg}$ projectile onto the nucleus of Comet 9P/Tempel 1 leading to an induced activity outburst of the nucleus. This provided the unique opportunity to directly observe fresh coma material ejected from the nucleus under non-steadystate conditions. Theoretical considerations have led to the suggestion that the dust ejected from the impact site has been 


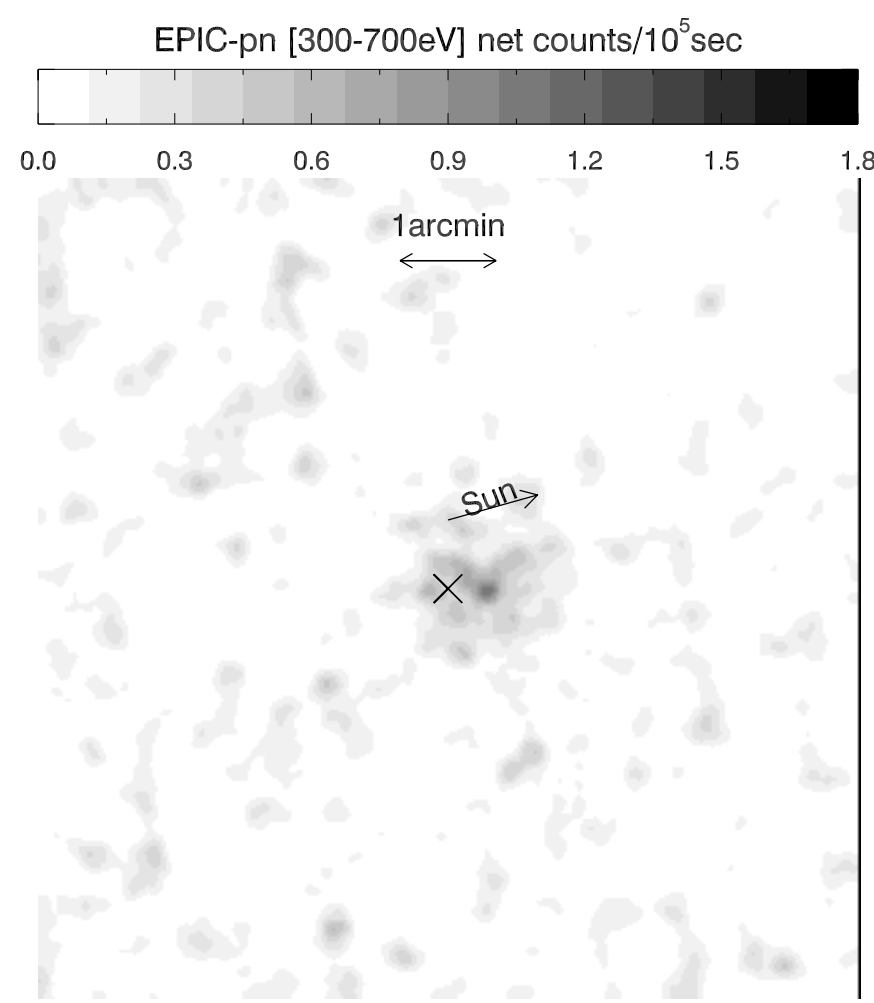

Fig. 1. Comet 9P/Tempel 1 observed with the EPIC instrument after the impact. X-rays were detected in the sunward hemisphere at a nucleus distance of $20000 \pm 6000 \mathrm{~km}$. The cross near the center marks the position of the comet nucleus. The projected Sun direction is also indicated. The image has been smoothed with an adaptive filter to enhance the weak signal.

broken up by the energetic impact process and thus has a different size distribution from the dust which is normally lifted off the surface by gentle sublimation processes (Hughes 2005). Here we present observations which provide direct evidence that icy grains were ejected from the comet nucleus as a result of the impact and that they subsequently disappeared due to evaporation of the icy component.

\section{Observations and data analysis}

Comet 9P/Tempel 1 was observed with the XMM-NEWTON OBSERVATORY $15.5 \mathrm{~h}$ before the impact, and again during and until $17.3 \mathrm{~h}$ after the impact with both, the X-ray instruments and the OPTICAL Monitor. The X-ray observations had to be integrated over the entire time spans before $(\approx 5 \mathrm{~h})$ and after impact $(\approx 15 \mathrm{~h})$ to reach a reasonable signal-to-noise ratio. The comet was detected in X-rays only after the impact as a weak X-ray source of a total flux of $\approx 3 \times 10^{-14} \mathrm{erg} \mathrm{cm}^{-2} \mathrm{~s}^{-1}$ at 300-700 eV. The EPIC image (Fig. 1) shows that the X-rays are not centered at the position of the comet nucleus, but some $20000 \mathrm{~km}$ away in the sunward hemisphere. If the X-ray detection is related to the material ejected from the nucleus after the impact, this implies an outflow velocity of $0.37 \pm 0.11 \mathrm{~km} \mathrm{~s}^{-1}$.

The OPTICAL MONITOR measured the evolution of photometric fluxes in 3 different filters with a time resolution of $2 \mathrm{~h}$. We used two filters in the ultraviolet (UVM2 and $U V W 1$ ) and a broadband $B$ filter in the visible spectral range. Figure 2

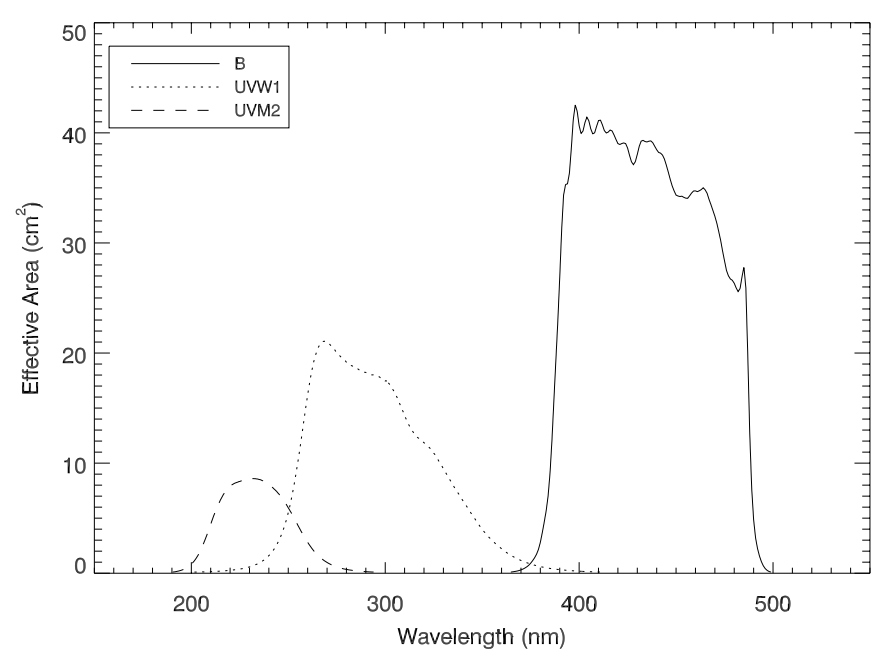

Fig. 2. The band passes and throughput curves for the $U V M 2$ (dashed), $U V W 1$ (dotted) and $B$ (solid) filter, folded with the OPTICAL MONITOR detector sensitivity.

shows the bandpasses and throughput curves for all three filters. The $U V W 1$ filter covers the $\mathrm{OH}(0-0)$ emission band of the $\left(A^{2} \Sigma^{+}-X^{2} \Pi\right)$ system centered at $308.6 \mathrm{~nm}$. As OH is a direct dissociation product of water, it is most frequently used to determine the water production rate in comets. The UVM2 filter measures the continuum light reflected by small coma grains at 200-270 nm and the $B$ filter covers a mixture of gas emission bands and continuum. The two-dimensional coma evolution in all 3 filters is depicted in Fig. 3. Before impact the comet could only be well detected in the $B$ filter, while there was no detection in $U V M 2$ (UV continuum) and only a faint detection in the $U V W 1(\mathrm{OH})$ filter. The immediate brightening of the coma resulting from the outburst induced by the impact and the subsequent decay in brightness over the next $17 \mathrm{~h}$ could be followed in all three filters. In $B$ and $U V W 1$, the comet remained visible until the end of the observing run and reached a spatial extension of about $6 \operatorname{arcsec}(3900 \mathrm{~km})$ approximately $1.2 \mathrm{~h}$ (in $U V W 1$ ) and $3 \mathrm{~h}$ (in $B$ ) after the impact. This coma diameter is consistent with nominal gas and dust outflow velocities of $\approx 0.5 \mathrm{~km} \mathrm{~s}^{-1}$ and $0.2 \mathrm{~km} \mathrm{~s}^{-1}$, respectively. In the UV continuum the comet was clearly detected only up to about $2 \mathrm{~h}$ after the impact, with a coma diameter of only about 1.8 arcsec $(1200 \mathrm{~km})$. Formally this would result in an outflow velocity of only $85 \mathrm{~m} / \mathrm{s}$. It is however very unlikely that the small grains reflecting at this wavelength $(\sim 230 \mathrm{~nm})$ had moved with such a small velocity, if at the same time larger dust particles represented in the $B$ filter (400-500 nm) moved more than twice as fast. We therefore conclude that the small grains reflecting in $U V M 2$ must have a very short lifetime, hence are destroyed before they reach nucleus distances beyond about $600 \mathrm{~km}$. If the outflow velocity of these grains is similar to that of the larger dust $\left(0.2 \mathrm{~km} \mathrm{~s}^{-1}\right)$, they have disintegrated within less than one hour.

\section{Detection of icy grains}

Figure 4 shows the photometric fluxes measured in all three filters as a function of time before and after impact, 


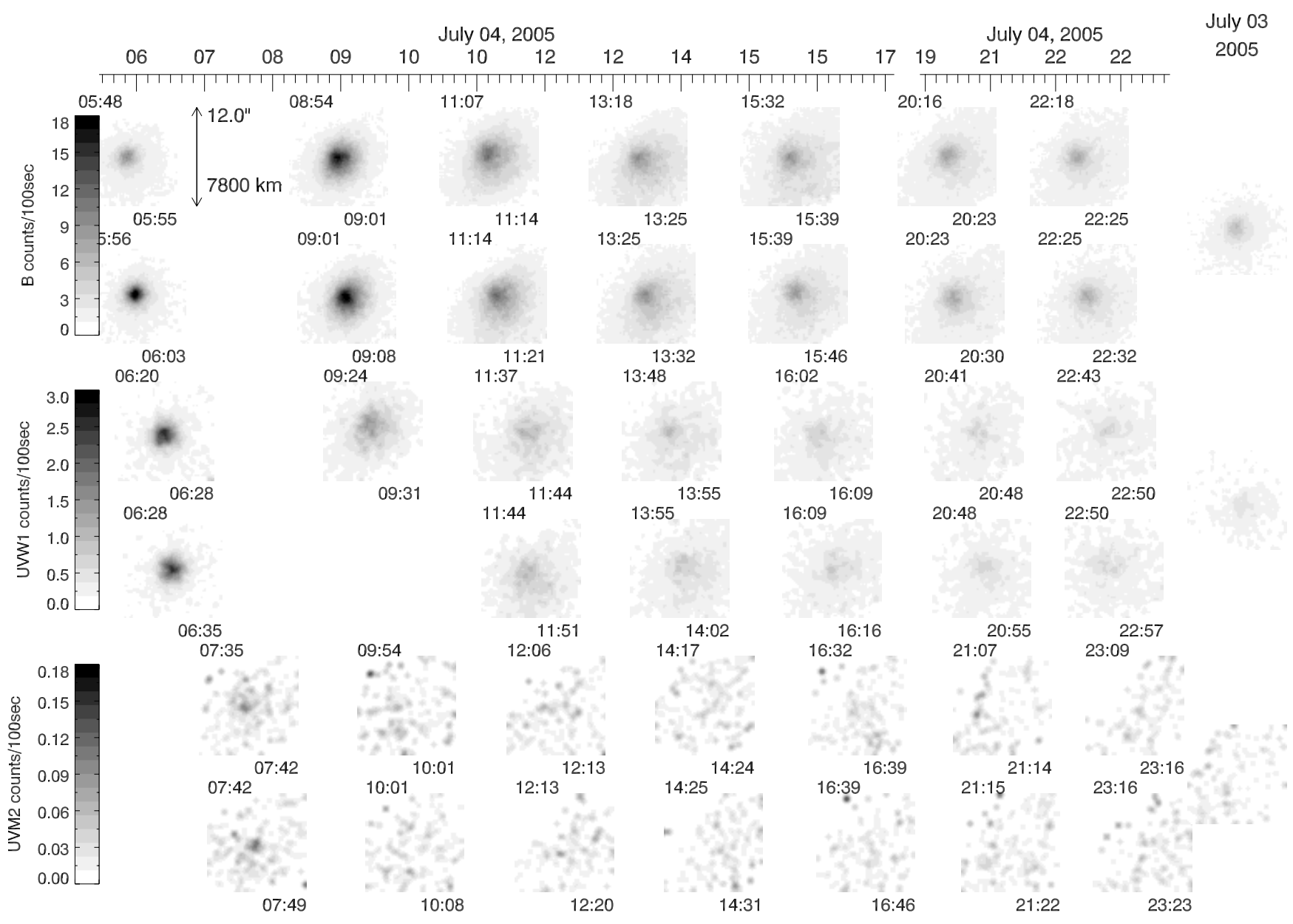

Fig. 3. The two-dimensional coma evolution observed as the result of the impact (impact time: 4 July 2005 at 5:52 UT). Before impact (right hand side images) the comet could be well detected only in the $B$ filter, while there is no detection in $U V M 2$ and only a faint detection in the $U V W 1$ filter. After impact the coma is however clearly detected in all three filters. In the $B$ and $U V W 1$ filter it remains visible for the next $8 \mathrm{~h}$ until the end of the observing run and the coma reached a spatial extension of about $6 \operatorname{arcsec}(3900 \mathrm{~km})$ approximately $1.2 \mathrm{~h}(U V W 1)$ and $3 \mathrm{~h}(B)$ after the impact. In the $U V M 2$ filter the comet was clearly detected only up to about $2 \mathrm{~h}$ after the impact, with a diameter of only about $1.8 \operatorname{arcsec}(1200 \mathrm{~km})$.

normalized to the pre-impact values. For each observation, the fluxes were integrated over a circular region of $6 \operatorname{arcsec}$ (equivalent to a coma diameter of $3900 \mathrm{~km}$ ). The light curve in each filter was fitted by the empirical function given in the figure and is only indicated to guide the eye. It is obvious that the evolution of the flux measured in the UV continuum is very different from the $\mathrm{OH}$ and broadband $B$. The light curves in $\mathrm{OH}$ and broadband $B$ both peak at about $3 \mathrm{~h}$ after the impact showing an increase in flux with respect to their pre-impact levels by factors of about 3 in the $\mathrm{OH}$ filter $(U V W 1)$ and 2.4 in the blue. They both decay at a similar pace, and when the comet became observable for ground-based telescopes about $16 \mathrm{~h}$ after the impact, they had reached the same flux levels as measured from ground. Observations of Comet 9P/Tempel 1 with the ESA OPTICAL GROUND STATION starting $16 \mathrm{~h}$ after the impact show a flux increase in the $B$ filter of a factor of 1.4 with regard to the pre-impact observations, which is consistent with the values obtained by the XMM-Newton Optical Monitor. In contrast, the light curve of the UV continuum (UVM2) peaks more than $1 \mathrm{~h}$ earlier and decreases at a much steeper pace than the other two, indicating that the small coma grains observed with this filter quickly disintegrated. Note, the first impact measurements with this filter were obtained about $1.6 \mathrm{~h}$ after the impact, when the maximum flux was already reached. The exact time of maximum can therefore not be extracted for this filter and it may even be that the light curve peaked immediately after the impact at an even higher level. For the $\mathrm{OH}$ and the $B$ filter on the other hand, measurements are available during and shortly after the impact allowing data point supported fits which confirm an increase and subsequent decrease phase for each of these two curves.

From the fact that after impact the light curves of $\mathrm{OH}$ and broadband $B$ peak at $>1.2 \mathrm{~h}$ after the UV continuum light curve we conclude that the small particles seen in the UV were icy grains that disintegrated, at least in part, by evaporating water (and probably also other gaseous species). It has been known for a long time that water is the most abundant volatile species in a comet and accounts for about $90 \%$ of its gas production rate. It is also well known that inside a heliocentric distance of $3 \mathrm{AU}$, water is the main driver of activity of a comet nucleus. Whether icy grains do or do not exist in the coma of a comet close to the Sun has therefore been of major importance for 


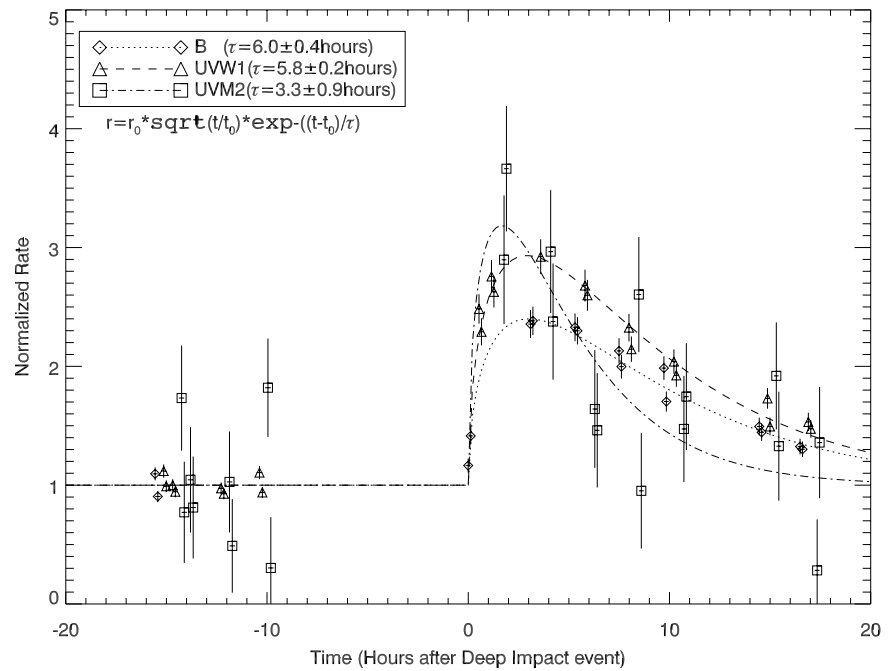

Fig. 4. The light curves measured in the UVM2 ( $\square), U V W 1(\triangle)$ and $B(\diamond)$ filter before, during and after the impact. The light curve in the UVM2 representing the rise and decay of small coma grains reaches its maximum more than $1 \mathrm{~h}$ before the light curves in $U V W 1$, representing the $\mathrm{OH}$ abundance and the $B$ filter covering a mixture of gas emission bands and continuum. The $U V M 2$ light curve also decays at a pace that is about twice a high as that of the other two curves. For each observation, the fluxes were integrated over a circular region of 6 arcsec (equivalent to a coma diameter of $3900 \mathrm{~km}$ ). This unfortunately increases the $1 \sigma$ error bars of the fluxes measured in the $U V M 2$ filter, but this area was selected to ensure that the spatial extension of the coma in the $B$ and $U V W 1$ filter is covered.

understanding the physical and chemical processes in the nearnucleus region of an active comet. However, as the lifetime of micron and sub-micron size icy grains is very short at heliocentric distances smaller than $2 \mathrm{AU}$, they have not been detectable by remote-sensing observations of a cometary coma, which is at steady-state conditions. Attempts have therefore been made to detect water ice in the coma of comets, which are active at large heliocentric distances. After a number of inconclusive searches, evidence for the presence of water ice was eventually found in infrared spectra obtained of comet Hale-Bopp (C/1995 O1) at heliocentric distances of 7 AU (Davies et al. 1997) and 2.9 AU (Lellouch et al. 1998). The observations of the DEEP IMPACT induced outburst of comet 9P/Tempel 1 provided the unique opportunity to characterize the properties of fresh cometray material coming off the comet nucleus under non-steady state conditions. Hughes (2005) demonstrated that the mass loss during and immediately after the impact was probably not in form of gentle water sublimation, which is consistent with the discovery reported here. Rapidly disintegrating small icy grains were detected shortly after the impact. The maximum abundance of these small grains was observed as early as $1.5 \mathrm{~h}$ after the impact, while larger particles reflecting in the visible spectral range reached their maximum only $1.5 \mathrm{~h}$ later. This proves the long standing assumption that the water in comets does not exclusively sublimate directly from the comet nucleus, but is also ejected in form of small icy grains at least during outbursts. As we have no measurements of the small grains at the time of the impact, we cannot determine whether they have reached their maximum abundance only when we took our first observation or earlier. For grains intrinsic to the nucleus this would be immediately after impact. If these icy grains came directly from the nucleus this would have strong implications for the formation of a comet nucleus and lead to constraints for the accretion models of the solar system.

\section{References}

Davies, J. K., Roush, T. L., Cruikshank, D. P., et al. 1997, Icarus, 127, 238

Hanner, M. S. 1981, Icarus, 47, 342

Huebner, W. F., \& Weigert, A. 1966, ZAp, 64, 185

Hughes, D. W. 2005, MNRAS, MN-05-0107

Lellouch, E., Crovisier, J., Lim, T., et al. 1998, A\&A, 339, L9

Whipple, F. L. 1950, ApJ, 111, 375 